\title{
Fourth supplement to the catalogue of observed periods of Ap stars
}

\author{
F.A. Catalano ${ }^{1}$ and P. Renson ${ }^{2}$ \\ 1 Istituto di Astronomia dell' Università and C.N.R.-G.N.A. Unità di Ricerca di Catania, Città Universitaria, I-95125 Catania, \\ Italia \\ e-mail: fcatalano@astrct.ct.astro.it \\ 2 Institut d'Astrophysique, Université de Liège, Avenue de Cointe 5, B-4000 Liège, Belgium \\ e-mail: u2148ap@vm1.ulg.ac.be
}

Received February 20; accepted May 9, 1996

\begin{abstract}
New data on the periods of Ap stars with references are presented (Table 1). 21 further stars are introduced for which a periodic variability has recently been discovered or for which it was known before but it was not reported in previous issues of this catalogue. For many stars also present in previous issues of the catalogue new determinations of the periods are given. Recently attributed variable star names are also quoted. Table 1, notes to this Table and related references are only available in LaTEX form at the CDS via anonymous ftp 130.79.128.5 (filename: DS1178.tex).
\end{abstract}

Key words: catalogs — stars: chemically peculiar — stars: variables: other

\section{Introduction}

Several new or improved data have appeared in the literature in the last three years to make the updating of the catalogue of observed periods of Ap stars useful.

Most of the results here reported do simply confirm or improve a previously known value of the period on the basis of new observations. This is very important for planning observations.

We also report here the variable star names which have been attributed since the publication of the previous update of our catalogue.

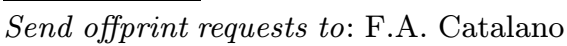

\section{The catalogue}

The presentation of the data is the same as that adopted in the main catalogue and the supplements (Catalano \& Renson 1984, 1988; Catalano et al. 1991, 1993).

The stars which appear here for the first time are marked with a + before their HD number: we present here 21 such stars.

As in the previous issues of the catalogue we have marked by asterisks the presence of notes; a double asterisk indicates a new or modified note. Notes are here reported for the newly introduced stars marked with an asterisk and for those marked with a double one. For the stars which were quoted in the previous issues (one asterisk), we do not report here the list of the notes: they can be easily found in Catalano \& Renson $(1984,1988)$ and in Catalano et al. (1991, 1993).

In Table 1 some stars are present without references: this means that a variable star name has been recently attributed or was not known to us before, or a new or a modified note has been introduced.

Finally we want to point out that the periods listed in the present supplement, as in the previous issues, are physically linked with nothing but axial rotation (via the oblique rotator model) or eclipses, hence we have not included the very short periods, i.e. those attributed to pulsations or oscillations.

Two stars among the newly introduced ones do not have any HD number (one of them has a HDE number); they are placed at the end of the Table, and their right ascensions and declinations are given in the notes.

Table 1, notes to this Table and related references are available in LaTEX form at the CDS via anonymous ftp 130.79.128.5 (filename: DS1178.tex).

We wish to ask our colleagues working on every aspect of Ap stars to send to F.A. Catalano or P. Renson their 
results. This would make easier the task of continuous updating the catalogue.

Acknowledgements. Our thanks are due to the colleagues who have kindly supplied us their results.

\section{References}

Catalano F.A., Renson P., 1984, A\&AS 55, 371

Catalano F.A., Renson P., 1988, A\&AS 72, 1

Catalano F.A., Renson P., Leone F., 1991, A\&AS 87, 59

Catalano F.A., Renson P., Leone F., 1993, A\&AS 98, 269 